\title{
ASE' 18
}

Proceedings of the 33rd ACM/IEEE International Conference on

\section{Automated Software Engineering}

Edited by:

Marianne Huchard, Christian Kästner, and Gordon Fraser

Sponsored by:

ACM SIGSOFT, ACM SIGAI, CNRS, IEEE-CS

Supported by:

Huawei, Berger Levrault, Mobioos, Toyota InfoTechnology Center, Région Occitanie, Inria, LIRMM, CNRS, University of Montpellier, Institut MinesTélécom (Mines Alès), Montpellier University of Excellence, Investissements d'avenir 


\section{The Association for Computing Machinery, Inc. 2 Penn Plaza, Suite 701 New York, NY 10121-0701}

Copyright $\odot 2018$ by the Association for Computing Machinery, Inc (ACM). Permission to make digital or hard copies of portions of this work for personal or classroom use is granted without fee provided that the copies are not made or distributed for profit or commercial advantage and that copies bear this notice and the full citation on the first page. Copyrights for components of this work owned by others than ACM must be honored. Abstracting with credit is permitted.

To copy otherwise, to republish, to post on servers or to redistribute to lists, requires prior specific permission and/or a fee. Request permission to republish from: Publications Dept. ACM, Inc.

Fax +1-212-869-0481 or E-mail permissions@acm.org.

For other copying of articles that carry a code at the bottom of the first or last page, copying is permitted provided that the per-copy fee indicated in the code is paid through the Copyright Clearance Center, 222 Rosewood Drive, Danvers, MA 01923.

\section{Notice to Authors of Past ACM-Published Articles}

ACM intends to create a complete electronic archive of all articles and/or other material previously published by ACM. If you have written a work that was previously published by ACM in any journal or conference proceedings prior to 1978, or any SIG Newsletter at any time, and you do NOT want this work to appear in the ACM Digital Library, please inform permissions@acm.org, stating the title of the work, the author(s), and where and when published.

ACM ISBN: 978-1-4503-5937-5

Additional copies may be ordered prepaid from:

$\begin{array}{ll}\text { ACM Order Department } & \text { (U.S.A. and Canada) } \\ \text { P.O. BOX 11405 } & +1-212-626-0500 \\ \text { Church Street Station } & \text { (All other countries) } \\ \text { New York, NY 10286-1405 } & \text { Fax: +1-212-944-1318 } \\ & \text { E-mail: acmhelp@acm.org }\end{array}$

Cover photo by Virginie Fèche

Production: Conference Publishing Consulting

D-94034 Passau, Germany, info@conference-publishing.com 


\section{Message from the Chairs}

It is our great pleasure and honor to welcome everyone to the 33rd IEEE/ACM International Conference on Automated Software Engineering (ASE 2018). The ASE conference series is the premier research forum for automated software engineering. Each year it brings together researchers and practitioners from academia and industry to discuss foundations, techniques, and tools for automated analysis, design, implementation, testing, and maintenance of software systems.

This publication contains the proceedings of the conference, which was held between September 3 and September 7, 2018 in Montpellier, France. The first two days were allocated to workshops, tutorials, and the doctoral symposium. The main conference was held from September 5 to September 7, and featured keynotes, technical, experience, new idea, journal first, and demo papers.

The conference technical program features the latest development in the area of automated software engineering, including (and not limited to) research on mobile development and testing, automated program synthesis and repair, verification \& validation, program analysis, security, and quality assurance for machine learning techniques. Overall, the purpose of ASE research is to support developers with automated solutions for the construction and evolution of modern software systems.

ASE 2018 was held on the CORUM conference center, and was organized by LIRMM (Laboratory of Informatics, Robotics, Microelectronics of Montpellier, CNRS and University of Montpellier), and LGI2P (IMT-Mines Alès), with the help of members from University of Toulouse, Bordeaux, ONERA and Université De Montréal (UDM). The University of Montpellier (UM) was created in 2015 by the merger of University Montpellier 1 and University Montpellier 2. UM welcomes 47,000 students and gathers teachers and researchers from science, technology, medicine and environmental sciences, to educational sciences, economics, management, law and political science. LIRMM is a joint research unit of the University of Montpellier (UM) and the National Center for Scientific Research (CNRS), which brings together more than 425 people including University full and associate professors, CNRS and Inria junior and senior scientists, staff engineers, support and administrative staff and around 150 $\mathrm{PhD}$ candidates and postdoctoral fellows. LIRMM research activities focus on modeling and designing various hardware (robots \& IC), designing and reengineering software systems and software product lines, as well as research activities on theoretical computer science, data science and AI. LIRMM contributes then on a wide range of scientific objectives with expertise on big data, artificial intelligence, knowledge representation, software engineering and programming languages, bioinformatics, image processing, algorithms and computation, robotics (collaborative, humanoid, mobile, surgical), mechanical design, control architecture, human-machine interaction, neuroprosthesis, micro-electronics, IC and biosensors design, smart systems. LIRMM is also at the core of the Montpellier University of Excellence (MUSE) whose the ambition is to have a great impact in the fields of agriculture, environment and health. LGI2P (Laboratory of computer engineering and production engineering) is composed of two teams, ISOE (Interoperable System and Organisation Engineering) and KID (Knowledge representation and Image analysis for Decision). LGI2P is a laboratory of IMT Mines Alès school, a graduate Institute of Engineering and Applied Science in Alès, which is now part of the national Institute Mines-Télécom. 
As a major highlight, ASE 2018 attracted three internationally renowned keynote speakers, whose talks feature a quite diverse set of topics. Specifically:

- Roberto di Cosmo (University Paris Diderot): "Software Heritage: collecting, preserving and sharing all our source code"

- Jane Cleland-Huang (University of Notre Dame): “Automated Requirements Engineering”

- Lauren Herckis (CMU): "Implementation Science for Software Engineering: Bridging the Gap Between Research and Practice"

In addition, ASE 2018 featured an IEEE CS Harlan Mills Award keynote address by Gail Murphy on "The Need for Context in Software Engineering".

For the technical research track, we received 386 submissions. Of these, 24 were desk-rejected or withdrawn by the authors, while 362 entered the reviewing process (315 technical papers, 13 experience papers, and 34 new ideas papers). Each paper received at least three reviews, on average four reviews, from members of the program committee.

For this year, ASE switched to an online-only program-committee meeting, in which all papers were discussed electronically in the HotCRP platform. This switch allowed to scale the program committee, reduce the individual review load, and save money, time, and carbon dioxide for not flying over 50 program committee members to one point on the planet. Program committee members wrote 1470 reviews and over 3100 comments in the discussion on the papers, often deliberating benefits and problems in depth. All program committee members engaged in the discussion. In several cases additional program-committee members were involved in the discussion. In addition to the reviewers, an additional program-committee member oversaw each discussion and summarized the discussion and outcome in a metareview.

As in previous years, ASE adopted a lightweight double-blind reviewing process, in which manuscripts were submitted as double-blind, and authors' identities were revealed to reviewers after reviews were uploaded on the submission system.

As the outcome of the online discussions, we accepted 80 contributions (overall acceptance rate of $21 \%)$ :

- 64 technical papers (20\% acceptance rate);

- 5 experience papers (33\% acceptance rate);

- 5 new idea papers (13\% acceptance rate);

- In addition, 6 technical papers were reclassified as new idea papers, of which all were accepted to be included in the proceedings.

Some of the above contributions were conditionally accepted, and therefore checked again by the program committee before the final acceptance.

We could not conclude the description of the reviewing process without giving a BIG THANK to all program committee members, as well as to additional reviewers (listed in the proceedings). With such 
a very large number of submissions, they had to carry out an enormous amount of work in a very short time (about nine weeks).

ASE again featured presentations of journal-first papers. We welcomed applications from authors of papers published (or accepted for publication) no earlier than January $1^{\text {st }}, 2017$ in a journal within the scope of ASE, including Automated Software Engineering (Springer), IEEE Transaction of Software Engineering (IEEE TSE), ACM Transactions on Software Engineering and Methodology (TOSEM), and Empirical Software Engineering (Springer). To be considered, papers should not be extensions of previous conference papers and should not be part of journal-first programs at other conferences. Four such papers have been selected and invited. The presentation of these papers has been allocated in sessions also featuring the other technical contributions mentioned above.

Besides presentations of technical papers, the program of ASE 2018 also included formal presentations of 16 demo papers, as well as hands-on demonstrations of the related tools. These papers were selected from 44 submissions (37\% acceptance rate). We would like to thank the tool demonstration chairs Julia Lawall and Darko Marinov.

ASE 2018 also featured four workshops, held before the main conference:

- 7th International Workshop on Software Mining (SOFTWAREMINING 2018). Organizers: David Lo, Ming Li and Xiaoyin Wang

- 1st International Workshop on Machine Learning and Software Engineering in Symbiosis (MASES 2018). Organizers: Gilles Perrouin, Maxime Cordy, Mathieu Acher and Xavier Devroey

- International Workshop on Advances in Mobile App Analysis (A-Mobile 2018). Organizers: Li Li, Guozhu Meng, Jacques Klein and Sam Malek

- 2nd International Workshop on Refactoring (IWoR 2018). Organizers: Ali Ouni, Marouane Kessentini and Mel O Cinneide

- We would like to thank the workshop chairs Houari Sahraoui and Chouki Tibermacine, as well as all the workshop organizers and authors.

ASE 2018 also offered four tutorials on topics such as theorem proving, test, software variability, and model-driven engineering:

- Automated Formal Proofs for Program Verification. Presenters: Guillaume Burel, David Delahaye, Olivier Hermant, and Étienne Prun.

- Let the CI Spot the Holes in Tested Code with the Descartes Tool. Presenters: Benoit Baudry, Vincent Massol, and Oscar Luis Vera Pérez.

- Re-Engineering Software Variability into Software Product Lines. Presenters: Mathieu Acher and Tewfik Ziadi.

- Model your Modeling Workbench, and Generate Code and Documentation from your Models. Presenters: Jacob Geisel.

We would like to thank the tutorial chairs Yamine Aït Ameur and David Delahaye for selecting the tutorials, as well as the tutorial presenters and their attendees. 
Furthermore, as per ASE tradition, the pre-conference featured a doctoral symposium organized by Abdelhak-Djamel Seriai and Virginie Wiels. The symposium was opened by a keynote speech by Benoit Baudry (KTH Royal Institute of Technology in Stockholm, Sweden). Then, eight doctoral students presented their ongoing work and benefited from the advice given by the panelists.

Numerous organizing committee members have helped in various ways to make the event a success. In particular, we would like to thank:

- The Sponsorship Chair Anne-Lise Courbis. ASE 2018 received generous contributions from many cooperating organizations and companies, which helped to keep the registration rates low;

- The Social Media chairs Jessie Carbonnel and Alexandre Le Borgne for proactively advertising the conference on Twitter and Facebook;

- The Communication, photos and publicity chairs Virginie Fèche, Christelle Urtado, Thomas Lambolais and Roland Ducournau who prepared the flyers, the advertisement on the mailing lists and took photos at the conference;

- The Local Arrangements Chairs Elisabeth Greverie, Mégane Miquel, Christophe Dony (tool demo local chair), Didier Parigot (Inria support);

- The Finance Chair / Treasurer Mégane Miquel;

- The Proceedings Chairs Clémentine Nebut, Christelle Urtado and Philippe Reitz for interacting with the proceedings production company and for their help with putting together these proceedings;

- The Web Chairs Sylvain Vauttier and Michel Meynard for keeping the ASE 2018 website always up-to-date;

- The Student Volunteer Chairs Hinde Bouziane and Floréal Morandat, as well as to all student volunteers.

Last but not least, we would like to thank our sponsors - please refer to the conference website (http://ase2018.com/sponsors) - for providing various kinds of support: Huawei, Mobioos, Berger Levrault, Toyota InfoTech, Région Occitanie, CNRS, Montpellier University of excellence (MUSE) Investissement d'avenir, University of Montpellier, LIRMM, IMT Mines Alès, Inria, IEEE, ACM, ACM SIGAI and ACM SIGSOFT.

Marianne Huchard, ASE 2018 General Chair

Gordon Fraser and Christian Kästner, ASE 2018 Program Co-chairs

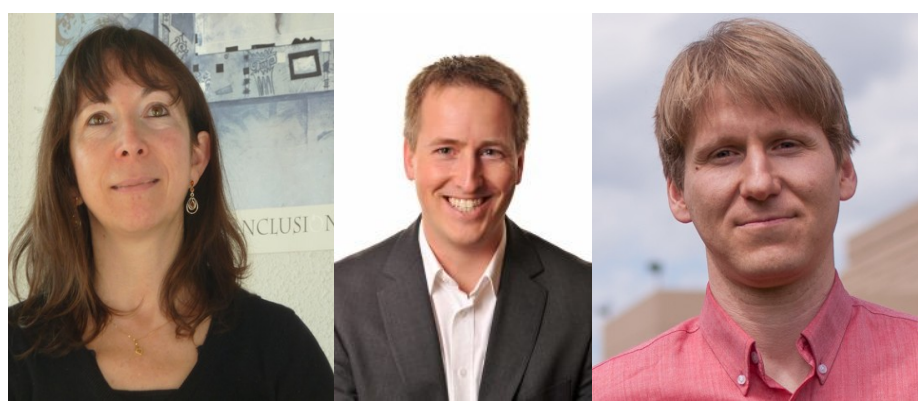




\section{ASE 2018 Organization}

Organizing Committee

\section{General Chair}

Marianne Huchard

Program Co-Chairs

Gordon Fraser

Christian Kästner

Tool Demonstration Chairs

Julia Lawall

Darko Marinov

Workshop Chairs

Houari Sahraoui

Chouki Tibermacine
University of Montpellier, France

University of Passau, Germany

Carnegie Mellon University, USA

Doctoral Symposium Chairs

Abdelhak-Djamel Seriai University of Montpellier, France

Virginie Wiels

Onera, France

\section{Tutorial Chairs}

Yamine Aït Ameur

INPT-ENSEEIHT/IRIT, France

David Delahaye

University of Montpellier, France

Journal-First Papers Chair

Bogdan Vasilescu

Carnegie Mellon University, USA

\section{Tool Demonstration Local Chair}

Christophe Dony

University of Montpellier, France

Publicity and Social Media Chairs

Jessie Carbonnel

Thomas Lambolais

University of Montpellier, France

Alexandre LeBorgne

IMT Mines Alès, France

Christelle Urtado

IMT Mines Alès, France

IMT Mines Alès, France

\section{Local Arrangements Chairs}

Christophe Dony

Elisabeth Greverie

University of Montpellier, France University of Montpellier, France

Mégane Miquel

Didier Parigot

Inria Sophia-Antipolis, France 
Sponsorship Chair

Anne-Lise Courbis

Finance Chairs/Treasurers

Elisabeth Greverie

Mégane Miquel

Proceedings Chairs

Clémentine Nebut

Philippe Reitz

Christelle Urtado

Student Volunteer Chairs

Hinde Bouziane

Floréal Morandat

Communication Chair

Virginie Fèche

Photo Chairs

Roland Ducournau

Virginie Fèche

Web Chairs

Michel Meynard

Sylvain Vauttier

\section{Steering Committee}

Paul Grünbacher

Michael R. Lowry

Robert J. Hall

Michael Goedicke

Houari Sahraoui

Bernd Fischer

Gabriele Taentzer

Tim Menzies

Ewen Denney

Tevfik Bultan

Myra Cohen

Mike Whalen

Lars Grunske

David Lo

Sven Apel

Sarfraz Khurshid

Marsha Chechik
IMT Mines Alès, France

University of Montpellier, France

University of Montpellier, France

University of Montpellier, France

University of Montpellier, France

IMT Mines Alès, France

University of Montpellier, France

Enseirb-Matmeca, France

University of Montpellier, France

University of Montpellier, France

University of Montpellier, France

University of Montpellier, France

IMT Mines Alès, France

Johannes Kepler University Linz, Austria

NASA Ames Research Center, USA

AT\&T Labs Research, USA

University of Duisburg-Essen, Germany

Université de Montréal, Canada

University of Stellenbosch, South Africa

Philipps-Universität Marburg, Germany

North Carolina State University, USA

Nasa Intelligent Systems Division, USA

University of California Santa Barbara, USA

University of Nebraska, USA

University of Minnesota, USA

Humboldt University, Berlin, Germany

Singapore Management University, Singapore

University of Passau, Germany

The University of Texas at Austin, USA

University of Toronto, Canada 
Grigore Rosu

Massimiliano di Penta

Tien Nguyen
University of Illinois, Urbana Champaign, USA

University of Sannio, Italy

University of Texas at Dallas, USA

\section{Program Committee - Research Track}

Mathieu Acher

Nada Amin

Sven Apel

Andrea Arcuri

Alberto Bacchelli

Hamid Bagheri

Earl T. Barr

Jonathan Bell

Thorsten Berger

Antonia Bertolino

Domenico Bianculli

Christian Bird

Eric Bodden

Tevfik Bultan

Yuanfang Cai

Yan Cai

Marsha Chechik

Jane Cleland-Huang

Myra Cohen

Elisabetta Di Nitto

Massimiliano Di Penta

Sebastian Erdweg

Robert Feldt

Antonio Filieri

Bernd Fischer

Juan P. Galeotti

Marie-Pierre Gervais

Milos Gligoric

Michael Goedicke

Alex Groce

John Grundy

Lars Grunske

Paul Grünbacher

Jianmei Guo

Yann-Gaël Guéhéneuc

Sonia Haiduc

Robert J. Hall

Dan Hao

Yue Jia

Brittany Johnson
University of Rennes / IRISA, France

University of Cambridge, UK

University of Passau, Germany

Westerdals, Norway, and Uni. Luxembourg, Luxembourg

University of Zurich, Switzerland

University of Nebraska-Lincoln, USA

University College London, UK

George Mason University, USA

Chalmers | University of Gothenburg, Sweden

CNR-ISTI, Italy

University of Luxembourg, Luxembourg

Microsoft Research, USA

Paderborn University \& Fraunhofer IEM, Germany

University of California, Santa Barbara, USA

Drexel University, USA

Institute of Software, Chinese Academy of Sciences, China

University of Toronto, Canada

University of Notre Dame, USA

University of Nebraska-Lincoln, USA

Politecnico di Milano, Italy

University of Sannio, Italy

TU Delft, Netherlands

Chalmers University of Technology, Sweden

Imperial College London, UK

Stellenbosch University, South Africa

Universidad de Buenos Aires, Argentina

University Paris Nanterre \& LIP6, France

The University of Texas at Austin, USA

University of Duisburg-Essen / paluno, Germany

Northern Arizona University, USA

Deakin University, Australia

Humboldt-Universität zu Berlin, Germany

Johannes Kepler University Linz, Austria

Alibaba Group, China

Concordia University, Canada

Florida State University, USA

AT\&T Labs Research, USA

Peking University, China

Facebook London \& UCL, UK

University of Massachusetts, Amherst, USA 
Christine Julien

Rene Just

Yasutaka Kamei

Aditya Kanade

Nicholas A. Kraft

Wei Le

Claire Le Goues

Yves Le Traon

Axel Legay

Yang Liu

David Lo

Shahar Maoz

Leonardo Mariani

Shane McIntosh

Na Meng

Ana Milanova

Mehdi Mirakhorli

Audris Mockus

Sarah Nadi

Tien N. Nguyen

ThanhVu (Vu) Nguyen

Klaus Ostermann

Chris Parnin

Liliana Pasquale

Xin Peng

John Penix

Andy Podgurski

Denys Poshyvanyk

Michael Pradel

Mukul Prasad

Baishakhi Ray

Romain Robbes

José Miguel Rojas

Abhik Roychoudhury

Julia Rubin

Neha Rungta

Anita Sarma

Federica Sarro

Koushik Sen

Elena Sherman

Norbert Siegmund

Matt Staats

Kathryn Stolee

Gabriele Taentzer

Lin Tan

Aditya V. Thakur
The University of Texas at Austin, USA

University of Massachusetts, Amherst, USA

Kyushu University, Japan

Indian Institute of Science, India

ABB Corporate Research, USA

Iowa State University, USA

Carnegie Mellon University, USA

University of Luxembourg, Luxembourg

Inria, France

Nanyang Technological University, China

Singapore Management University, Singapore

Tel Aviv University, Israel

University of Milano Bicocca, Italy

McGill University, Canada

Virginia Tech, USA

Rensselaer Polytechnic Institute, USA

Rochester Institute of Technology, USA

The University of Tennessee, USA

University of Alberta, Canada

University of Texas at Dallas, USA

University of Nebraska, Lincoln, USA

University of Tübingen, Germany

North Carolina State University, USA

University College Dublin \& Lero, Ireland

Fudan University, China

Google, Inc., USA

Case Western Reserve University, USA

College of William and Mary, USA

TU Darmstadt, Germany

Fujitsu Laboratories of America, USA

University Of Virginia, USA

Free University of Bozen-Bolzano, Italy

University of Leicester, UK

National University of Singapore, Singapore

University of British Columbia, Canada

Amazon Web Services, USA

Oregon State University, USA

University College London, UK

UC Berkeley, USA

Boise State University, USA

Bauhaus-University Weimar, Germany

North Carolina State University, USA

Philipps-Universität Marburg, Germany

University of Waterloo, Canada

University of California, Davis, USA 
Paolo Tonella

Bogdan Vasilescu

Willem Visser

Neil Walkinshaw

Michael Whalen

Zhenchang Xing

Yingfei Xiong

Shin Yoo

Tingting Yu

Lingming Zhang

Charles Zhang

Xiangyu Zhang

Hongyu Zhang

Hao Zhong

Lukasz Ziarek

Andrea Zisman
Fondazione Bruno Kessler, Italy

Carnegie Mellon University, USA

Stellenbosch University, South Africa

University of Leicester, UK

University of Minnesota, USA

Australian National University, Australia

Peking University, China

Korea Advanced Institute of Science and Technology, S. Korea

University of Kentucky, USA

The University of Texas at Dallas, USA

HKUST \& SourceBrella, China

Purdue University, USA

The University of Newcastle, Australia

Shanghai Jiao Tong University, China

SUNY at Buffalo, USA

The Open University, UK

\section{Additional Reviewers - Research Track}

Khaled E. Ahmed

Afnan Al-Subaihin

Alexander Altman

Hlib Babii

Oliver Bračevac

Will Byrd

Michael Cao

Andrew Gacek

Srishti Gaihre

Cuiyun Gao

Michele Guerriero

Daco Harkes

Andrea Janes

Harshavardhan Kadiyala

Moein Owhadi Kareshk

Sven Keidel

Stephen Kell

Sahar Kokaly

Jeremy Lacomis

Duling Lai

Sihan Li

Yi Li

Mehran Mahmoudi

George Mathew

Federico Mora

Catherine Lee

Fernando Lopez de la Mora
University of British Columbia, Canada

University College London, UK

University of Illinois, Urbana-Champaign, USA

Free University of Bozen-Bolzano, Italy

TU Darmstadt, Germany

University of Alabama at Birmingham, USA

University of British Columbia, Canada

Amazon Web Services, USA

Free University of Bozen-Bolzano, Italy

The Chinese University of Hong Kong, China

Politecnico di Milano, Italy

TU Delft, Netherlands

Free University of Bozen-Bolzano, Italy

University of British Columbia, Canada

University of Alberta, USA

TU Delft, Netherlands

University of Cambridge, UK

University of Toronto, USA

Carnegie Mellon University, USA

University of British Columbia, Canada

University of Illinois at Urbana-Champaign, USA

University of Toronto, Canada

University of Alberta, USA

North Carolina State University, USA

University of Toronto, Canada

University of British Columbia, Canada

University of Alberta, Canada 


$\begin{array}{ll}\text { Thibaud Lutellier } & \text { University of Waterloo, Canada } \\ \text { Pengyu Nie } & \text { The University of Texas at Austin, USA } \\ \text { Benyamin Noori } & \text { University of Alberta, Canada } \\ \text { Matheus Paixao } & \text { University College London, UK } \\ \text { Karl Palmskog } & \text { The University of Texas at Austin, USA } \\ \text { Huilian Qiu } & \text { Carnegie Mellon University, USA } \\ \text { John Regehr } & \text { University of Utah, USA } \\ \text { Ripon K. Saha } & \text { Fujitsu Laboratories of America, USA } \\ \text { Rick Salay } & \text { University of Toronto, Canada } \\ \text { Satish Sarraf } & \text { University of British Columbia, Canada } \\ \text { Ramy Shahin } & \text { University of Toronto, Canada } \\ \text { Tamás Szabó } & \text { TU Delft / itemis AG, Netherlands } \\ \text { Michael Tautschnig } & \text { Queen Mary University of London, UK } \\ \text { Sebastian Uchitel } & \text { Imperial College and University of Buenos Aires, Argentina } \\ \text { Marat Valiev } & \text { Carnegie Mellon University, USA } \\ \text { Peipei Wang } & \text { North Carolina State University, USA } \\ \text { Yingying Wang } & \text { University of British Columbia, Canada } \\ \text { David Widder } & \text { Carnegie Mellon University, USA } \\ \text { Edmund Wong } & \text { University of Waterloo, Canada } \\ \text { Hiroaki Yoshida } & \text { Fujitsu Laboratories of America, USA } \\ \text { Junbin Zhang } & \text { University of British Columbia, Canada } \\ \text { Chenguang Zhu } & \text { The University of Texas at Austin, USA } \\ & \end{array}$

\section{Program Committee - Tool Demonstrations}

Nazareno Aguirre

Tegawendé F. Bissyandé

Marcel Böhme

Yuanfang Cai

Shing-Chi Cheung

Robert Dyer

Jean-Rémy Falleri

Jeff Gray

Raghavan Komondoor

Tien N. Nguyen

Christoph Reichenbach

Steven P. Reiss

Romain Rouvoy

Nastaran Shafiei

Elena Sherman

Sara Sprenkle

Nikolai Tillmann

Ye Yang

Vadim Zaytsev

Jianjun Zhao

Minghui Zhou
University of Rio Cuarto and CONICET, Argentina

SnT, University of Luxembourg, Luxembourg

Monash University, Australia

Drexel University, USA

The Hong Kong University of Science and Technology, China

Bowling Green State University, USA

Bordeaux INP, France

University of Alabama, USA

Indian Institute of Science, India

University of Texas - Dallas, USA

Lund University, Sweden

Brown University, USA

Univ. Lille / Inria, France

NASA Ames Research Center/SGT, USA

Boise State University, USA

Washington and Lee University, USA

Facebook, USA

Stevens Institute of Technology, USA

Raincode Labs, Belgium

Kyushu University, Japan

Peking University, China 


\section{Program Committee - Doctoral Symposium}

Aldeida Aleti

Mireille Blay-Fornarino

Massimiliano Di Penta

Yann-Gaël Guéhéneuc

Foutse Khomh
Monash University, Australia

Nice University, France

University of Sannio, Italy

Concordia University, Canada

Polytechnique Montreal, Canada 
GOLD SPONSOR

\section{SE HUAWEI}

SILVER SPONSORS

Berger

LevPault

\section{nilobioos}

BRONZE SPONSOR

$$
\begin{aligned}
& \text { TOYOTA } \\
& \text { INFOTECH }
\end{aligned}
$$

TECHNICAL SPONSORS
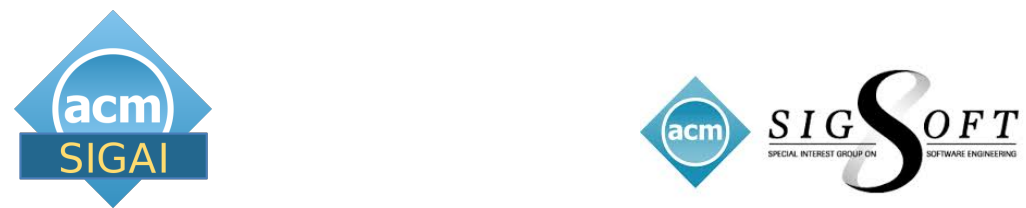

- IEEE

INSTITUTIONAL SPONSORS
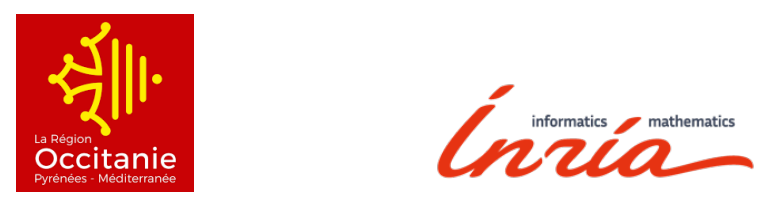

BLIRMM

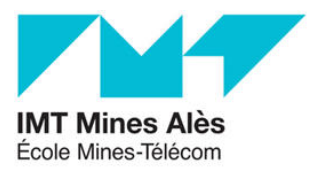

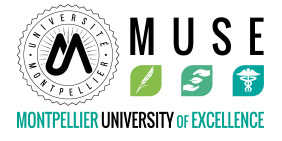

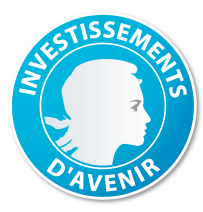




\section{Contents}

\section{Frontmatter}

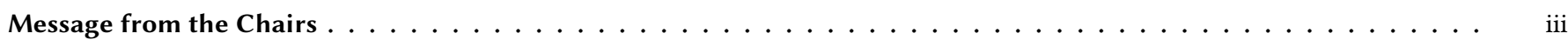

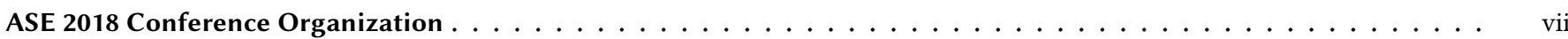

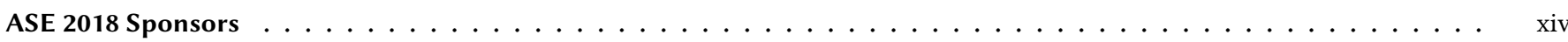

\section{Keynotes}

Software Heritage: Collecting, Preserving, and Sharing All Our Source Code (Keynote)

Roberto Di Cosmo - Inria, France; University Paris Diderot, France . . . . . . . . . . . . . . . . . . . . . .

Automated Requirements Engineering Challenges with Examples from Small Unmanned Aerial Systems (Keynote) Jane Cleland-Huang - University of Notre Dame, USA . . . . . . . . . . . . . . . . . . . . . . . . . . . . . .

Implementation Science for Software Engineering: Bridging the Gap between Research and Practice (Keynote) Lauren Herckis - Carnegie Mellon University, USA ． . . . . . . . . . . . . . . . . . . . . . . . .

The Need for Context in Software Engineering (IEEE CS Harlan Mills Award Keynote)

Gail C. Murphy - University of British Columbia, Canada . . . . . . . . . . . . . . . . . . . . . . . .

\section{Technical Research Papers}

\section{Performance}

On Adopting Linters to Deal with Performance Concerns in Android Apps

Sarra Habchi, Xavier Blanc, and Romain Rouvoy - Inria, France; University of Lille, France; University of Bordeaux, France . . . .

PerfLearner: Learning from Bug Reports to Understand and Generate Performance Test Frames

Xue Han, Tingting Yu, and David Lo - University of Kentucky, USA; Singapore Management University, Singapore . . . . . . . . .

AutoConfig: Automatic Configuration Tuning for Distributed Message Systems

Liang Bao, Xin Liu, Ziheng Xu, and Baoyin Fang - Xidian University, China; University of California at Davis, USA . . . . . . . .

Is This Class Thread-Safe? Inferring Documentation using Graph-Based Learning

Andrew Habib and Michael Pradel - TU Darmstadt, Germany . . . . . . . . . . . . . . . . . . . . . .

\section{Testing Studies}

A Large-Scale Study of Test Coverage Evolution

Michael Hilton, Jonathan Bell, and Darko Marinov - Carnegie Mellon University, USA; George Mason University, USA; University of Illinois at Urbana-Champaign, USA . . . . . . . . . . . . . . . . . . . . . . . . . . . .

Effectiveness and Challenges in Generating Concurrent Tests for Thread-Safe Classes

Valerio Terragni and Mauro Pezzè - University of Lugano, Switzerland . . . . . . . . . . . . . . . . . . . . . . . .

\section{Build and Test Automation}

Scalable Incremental Building with Dynamic Task Dependencies Gabriël Konat, Sebastian Erdweg, and Eelco Visser - Delft University of Technology, Netherlands . . . . . . . . . . . . . . . . . .

Noise and Heterogeneity in Historical Build Data: An Empirical Study of Travis CI Keheliya Gallaba, Christian Macho, Martin Pinzger, and Shane McIntosh - McGill University, Canada; University of Klagenfurt,

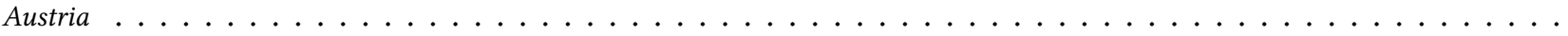

\section{Quality Assurance for Machine Learning Techniques}

\section{Automated Directed Fairness Testing} Sakshi Udeshi, Pryanshu Arora, and Sudipta Chattopadhyay - Singapore University of Technology and Design, Singapore; BITS Pilani,

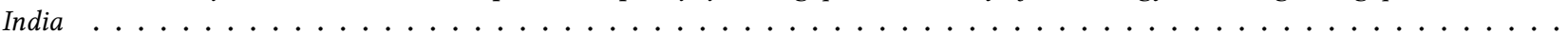

Concolic Testing for Deep Neural Networks Youcheng Sun, Min Wu, Wenjie Ruan, Xiaowei Huang, Marta Kwiatkowska, and Daniel Kroening - University of Oxford, UK; University of Liverpool,$U K \ldots \ldots \ldots \ldots \ldots$ 
DeepGauge: Multi-Granularity Testing Criteria for Deep Learning Systems

Lei Ma, Felix Juefei-Xu, Fuyuan Zhang, Jiyuan Sun, Minhui Xue, Bo Li, Chunyang Chen, Ting Su, Li Li, Yang Liu, Jianjun Zhao, and Yadong Wang - Harbin Institute of Technology, China; Nanyang Technological University, Singapore; Carnegie Mellon University, USA; Kyushu University, Japan; University of Illinois at Urbana-Champaign, USA; Monash University, Australia . . . . . . . . . . . . . .

DeepRoad: GAN-Based Metamorphic Testing and Input Validation Framework for Autonomous Driving Systems Mengshi Zhang, Yuqun Zhang, Lingming Zhang, Cong Liu, and Sarfraz Khurshid - University of Texas at Austin, USA; Southern University of Science and Technology, China; University of Texas at Dallas, USA . . . . . . . . . . . . . . . . . . . . . . .

\section{Variability}

Testing Autonomous Cars for Feature Interaction Failures using Many-Objective Search

Raja Ben Abdessalem, Annibale Panichella, Shiva Nejati, Lionel C. Briand, and Thomas Stifter - University of Luxembourg, Luxembourg; Delft University of Technology, Netherlands; IEE, Luxembourg ～. . . . . . . . . . . . . . . . . . . . . . .

Tackling Combinatorial Explosion: A Study of Industrial Needs and Practices for Analyzing Highly Configurable Systems Mukelabai Mukelabai, Damir Nešić, Salome Maro, Thorsten Berger, and Jan-Philipp Steghöfer - Chalmers University of Technology, Sweden; University of Gothenburg, Sweden; KTH, Sweden ～. . . . . . . . . . . . . . . . . . .

Understanding and Detecting Evolution-Induced Compatibility Issues in Android Apps Dongjie He, Lian Li, Lei Wang, Hengjie Zheng, Guangwei Li, and Jingling Xue - Institute of Computing Technology at Chinese Academy of Sciences, China; University of Chinese Academy of Sciences, China; UNSW, Australia . . . . . . . . . . . . . . . . . .

\section{Mining and Crowd Sourcing}

Characterizing the Natural Language Descriptions in Software Logging Statements Pinjia He, Zhuangbin Chen, Shilin He, and Michael R. Lyu - Chinese University of Hong Kong, China . . . . . . . . . . . . .

Assessing the Type Annotation Burden John-Paul Ore, Sebastian Elbaum, Carrick Detweiler, and Lambros Karkazis - University of Nebraska-Lincoln, USA . . . . . . . .

Mining File Histories: Should We Consider Branches? Vladimir Kovalenko, Fabio Palomba, and Alberto Bacchelli - Delft University of Technology, Netherlands; University of Zurich,

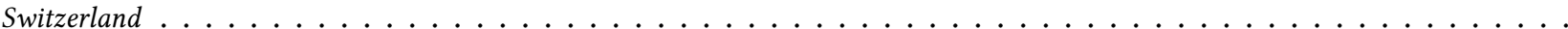

Tell Them Apart: Distilling Technology Differences from Crowd-Scale Comparison Discussions Yi Huang, Chunyang Chen, Zhenchang Xing, Tian Lin, and Yang Liu - Australian National University, Australia; Monash University, Australia; Nanyang Technological University, Singapore . . . . . . . . . . . . . . . . . . . . . . . .

\section{Security}

ReScue: Crafting Regular Expression DoS Attacks

Yuju Shen, Yanyan Jiang, Chang Xu, Ping Yu, Xiaoxing Ma, and Jian Lu - Nanjing University, China . . . . . . . . . . . .

TDroid: Exposing App Switching Attacks in Android with Control Flow Specialization Jie Liu, Diyu Wu, and Jingling Xue $-U N S W$, Australia $\ldots \ldots \ldots \ldots$

Model-Driven Run-Time Enforcement of Complex Role-Based Access Control Policies Ameni Ben Fadhel, Domenico Bianculli, and Lionel C. Briand - University of Luxembourg, Luxembourg . . . . . . . . . . . .

ContractFuzzer: Fuzzing Smart Contracts for Vulnerability Detection Bo Jiang, Ye Liu, and W. K. Chan - Beihang University, China; City University of Hong Kong, China . . . . . . . . . . . . . . . . .

\section{Developer Tools}

SEEDE: Simultaneous Execution and Editing in a Development Environment Steven P. Reiss, Qi Xin, and Jeff Huang - Brown University, USA ～. . . . . . . . . . . . . . . . . . . .

Effective API Recommendation without Historical Software Repositories Xiaoyu Liu, LiGuo Huang, and Vincent $\mathrm{Ng}$ - Southern Methodist University, USA; University of Texas at Dallas, USA . . . . . . . .

API Method Recommendation without Worrying about the Task-API Knowledge Gap Qiao Huang, Xin Xia, Zhenchang Xing, David Lo, and Xinyu Wang - Zhejiang University, China; Monash University, Australia; Australian National University, Australia; Singapore Management University, Singapore ～. . . . . . . . . . . . . . . . .

An Automated Approach to Estimating Code Coverage Measures via Execution Logs Boyuan Chen, Jian Song, Peng Xu, Xing Hu, and Zhen Ming (Jack) Jiang - York University, Canada; Baidu, China . . . . . . . . .

\section{Static Analysis}

How Many of All Bugs Do We Find? A Study of Static Bug Detectors Andrew Habib and Michael Pradel - TU Darmstadt, Germany . . . . . . . . . . . . . . . . . . . . . . . 
TRIMMER: Application Specialization for Code Debloating

Hashim Sharif, Muhammad Abubakar, Ashish Gehani, and Fareed Zaffar - University of Illinois at Urbana-Champaign, USA; Lahore

University of Management Sciences, Pakistan; SRI International, USA ～. . . . . . . . . . . . . . . . . . . . .

A Unified Lattice Model and Framework for Purity Analyses

Dominik Helm, Florian Kübler, Michael Eichberg, Michael Reif, and Mira Mezini - TU Darmstadt, Germany . . . . . . . . . . .

\section{Verification 1}

Control Flow-Guided SMT Solving for Program Verification

Jianhui Chen and Fei He - Tsinghua University, China . . . . . . . . . . . . . . . . . . . . . . . . .

PaMpeR: Proof Method Recommendation System for Isabelle/HOL

Yutaka Nagashima and Yilun He - Czech Technical University, Czechia; University of Innsbruck, Austria; University of Sydney, Australia

\section{Maintenance and Machine Learning}

Neural-Machine-Translation-Based Commit Message Generation: How Far Are We?

Zhongxin Liu, Xin Xia, Ahmed E. Hassan, David Lo, Zhenchang Xing, and Xinyu Wang - Zhejiang University, China; Monash University, Australia; Queen's University, Canada; Singapore Management University, Singapore; Australian National University, Australia . . .

Deep Learning Based Feature Envy Detection

Hui Liu, Zhifeng Xu, and Yanzhen Zou - Beijing Institute of Technology, China; Peking University, China . . . . . . . . . . . . .

Improving Automatic Source Code Summarization via Deep Reinforcement Learning

Yao Wan, Zhou Zhao, Min Yang, Guandong Xu, Haochao Ying, Jian Wu, and Philip S. Yu - Zhejiang University, China; Chinese Academy of Sciences, China; University of Technology Sydney, Australia; University of Illinois at Chicago, USA . . . . . . . . . . . .

\section{Symbolic Execution}

Template-Guided Concolic Testing via Online Learning Sooyoung Cha, Seonho Lee, and Hakjoo Oh - Korea University, South Korea . . . . . . . . . . . . . . . . . . .

Android Testing via Synthetic Symbolic Execution

Xiang Gao, Shin Hwei Tan, Zhen Dong, and Abhik Roychoudhury - National University of Singapore, Singapore; Southern University of Science and Technology, China . . . . . . . . . . . . . . . . . . . . . . . . . . . .

PARTI: A Multi-interval Theory Solver for Symbolic Execution

Oscar Soria Dustmann, Klaus Wehrle, and Cristian Cadar - RWTH Aachen University, Germany; Imperial College London, UK . .

\section{Maintenance}

Client-Specific Equivalence Checking

Federico Mora, Yi Li, Julia Rubin, and Marsha Chechik - University of Toronto, Canada; University of British Columbia, Canada . .

Replay without Recording of Production Bugs for Service Oriented Applications

Nipun Arora, Jonathan Bell, Franjo Ivančić, Gail Kaiser, and Baishakhi Ray - Dropbox, USA; George Mason University, USA; Google,

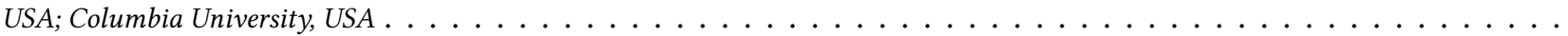

Reducing Interactive Refactoring Effort via Clustering-Based Multi-objective Search Vahid Alizadeh and Marouane Kessentini - University of Michigan, USA . . . . . . . . . . . . . . . . . .

\section{Software Quality}

FairFuzz: A Targeted Mutation Strategy for Increasing Greybox Fuzz Testing Coverage Caroline Lemieux and Koushik Sen - University of California at Berkeley, USA . . . . . . . . . . . . . . . . . . .

Efficiently Manifesting Asynchronous Programming Errors in Android Apps Lingling Fan, Ting Su, Sen Chen, Guozhu Meng, Yang Liu, Lihua Xu, and Geguang Pu - East China Normal University, China; Nanyang Technological University, Singapore; Chinese Academy of Sciences, China; New York University Shanghai, China . . . . . . . . . . .

Expandable Group Identification in Spreadsheets

Wensheng Dou, Shi Han, Liang Xu, Dongmei Zhang, and Jun Wei - University of Chinese Academy of Sciences, China; Institute of Software at Chinese Academy of Sciences, China; Microsoft Research, China . . . . . . . . . . . . . . . . . . .

Break the Dead End of Dynamic Slicing: Localizing Data and Control Omission Bug Yun Lin, Jun Sun, Lyly Tran, Guangdong Bai, Haijun Wang, and Jinsong Dong - National University of Singapore, Singapore; Singapore University of Technology and Design, Singapore; Singapore Institute of Technology, Singapore; Nanyang Technological University,

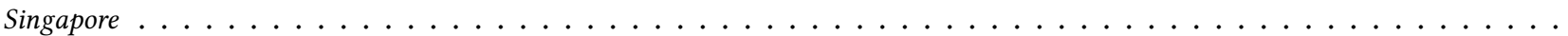




\section{Architecture and Requirements}

A Genetic Algorithm for Goal-Conflict Identification

Renzo Degiovanni, Facundo Molina, Germán Regis, and Nazareno Aguirre - National University of Río Cuarto, Argentina; CONICET,

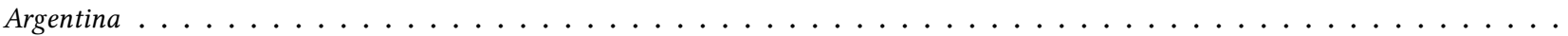

\section{Mobile Analysis}

Understanding and Detecting Callback Compatibility Issues for Android Applications

Huaxun Huang, Lili Wei, Yepang Liu, and Shing-Chi Cheung - Hong Kong University of Science and Technology, China; Southern

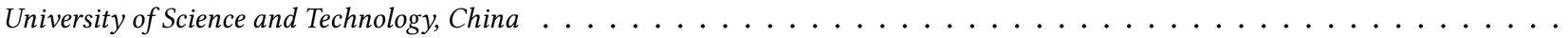

Detecting and Summarizing GUI Changes in Evolving Mobile Apps

Kevin Moran, Cody Watson, John Hoskins, George Purnell, and Denys Poshyvanyk - College of William and Mary, USA . . . . .

Empirically Assessing Opportunities for Prefetching and Caching in Mobile Apps

Yixue Zhao, Paul Wat, Marcelo Schmitt Laser, and Nenad Medvidović - University of Southern California, USA . . . . . . . . .

Safe Stream-Based Programming with Refinement Types

Benno Stein, Lazaro Clapp, Manu Sridharan, and Bor-Yuh Evan Chang - University of Colorado Boulder, USA; Uber Technologies, USA

\section{Repair}

Automated Model Repair for Alloy

Kaiyuan Wang, Allison Sullivan, and Sarfraz Khurshid - University of Texas at Austin, USA . . . . . . . . . . . . . . .

PFix: Fixing Concurrency Bugs Based on Memory Access Patterns

Huarui Lin, Zan Wang, Shuang Liu, Jun Sun, Dongdi Zhang, and Guangning Wei - Tianjin University, China; Singapore University of

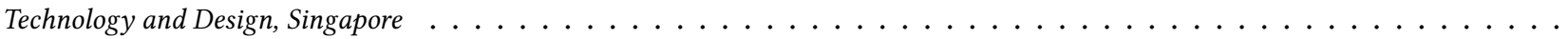

Generating Reusable Web Components from Mockups

Mohammad Bajammal, Davood Mazinanian, and Ali Mesbah - University of British Columbia, Canada . . . . . . . . . . . . .

Semantic Crash Bucketing

Rijnard van Tonder, John Kotheimer, and Claire Le Goues - Carnegie Mellon University, USA . . . . . . . . . . . . . . . .

\section{Verification 2}

A Symbolic Model Checking Approach to the Analysis of String and Length Constraints

Hung-En Wang, Shih-Yu Chen, Fang Yu, and Jie-Hong R. Jiang - National Taiwan University, Taiwan; National Chengchi University,

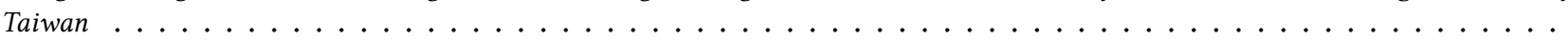

Domain-Independent Multi-threaded Software Model Checking

Dirk Beyer and Karlheinz Friedberger - LMU Munich, Germany . . . . . . . . . . . . . . . . . . . . .

Scheduling Constraint Based Abstraction Refinement for Weak Memory Models

Liangze Yin, Wei Dong, Wanwei Liu, and Ji Wang - National University of Defense Technology, China . . . . . . . . . . . . . .

\section{Code Differencing and Merging}

Datalog-Based Scalable Semantic Diffing of Concurrent Programs

Chungha Sung, Shuvendu K. Lahiri, Constantin Enea, and Chao Wang - University of Southern California, USA; Microsoft Research,

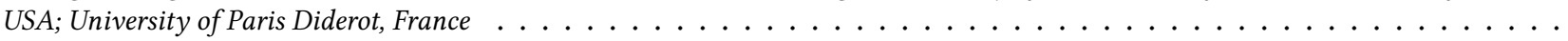

$\alpha$ Diff: Cross-Version Binary Code Similarity Detection with DNN

Bingchang Liu, Wei Huo, Chao Zhang, Wenchao Li, Feng Li, Aihua Piao, and Wei Zou - Institute of Information Engineering at Chinese Academy of Sciences, China; University of Chinese Academy of Sciences, China; Tsinghua University, China . . . . . . . . . . . . .

CIDiff: Generating Concise Linked Code Differences

Kaifeng Huang, Bihuan Chen, Xin Peng, Daihong Zhou, Ying Wang, Yang Liu, and Wenyun Zhao - Fudan University, China; Nanyang

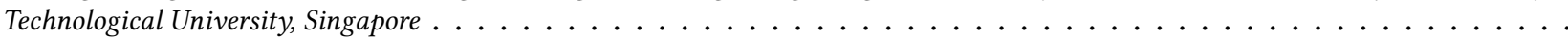

\section{Mobile Security}

Characterizing and Identifying Misexposed Activities in Android Applications Jiwei Yan, Xi Deng, Ping Wang, Tianyong Wu, Jun Yan, and Jian Zhang - Institute of Software at Chinese Academy of Sciences, China;

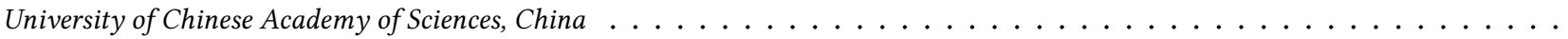

A Tale of Two Cities: How WebView Induces Bugs to Android Applications

Jiajun Hu, Lili Wei, Yepang Liu, Shing-Chi Cheung, and Huaxun Huang - Hong Kong University of Science and Technology, China;

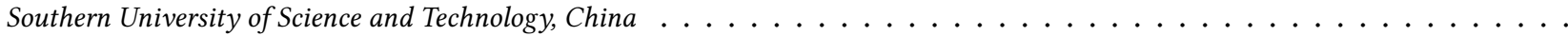


Dual-Force: Understanding WebView Malware via Cross-Language Forced Execution

Zhenhao Tang, Juan Zhai, Minxue Pan, Yousra Aafer, Shiqing Ma, Xiangyu Zhang, and Jianhua Zhao - Nanjing University, China;

Purdue University, USA . . . . . . . . . . . . . . . . . . . . . . . . . . . .

Self-Protection of Android Systems from Inter-component Communication Attacks

Mahmoud Hammad, Joshua Garcia, and Sam Malek - University of California at Irvine, USA . . . . . . . . . . . . . . .

\section{Experience Papers}

An Empirical Study of Android Test Generation Tools in Industrial Cases

Wenyu Wang, Dengfeng Li, Wei Yang, Yurui Cao, Zhenwen Zhang, Yuetang Deng, and Tao Xie - University of Illinois at Urbana-

Champaign, USA; University of Texas at Dallas, USA; Tencent, China . . . . . . . . . . . . . . . . .

Achieving Test Automation with Testers without Coding Skills: An Industrial Report

Davrondzhon Gafurov, Arne Erik Hurum, and Martin Markman - Norwegian Directorate of eHealth, Norway . . . . . . . . . . .

Navigating the Maze: The Impact of Configurability in Bioinformatics Software

Mikaela Cashman, Myra B. Cohen, Priya Ranjan, and Robert W. Cottingham - University of Nebraska-Lincoln, USA; Oak Ridge

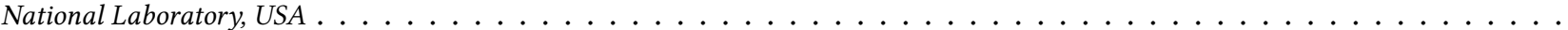

Automatically Testing Implementations of Numerical Abstract Domains Alexandra Bugariu, Valentin Wüstholz, Maria Christakis, and Peter Müller - ETH Zurich, Switzerland; MPI-SWS, Germany . . . .

Experiences Applying Automated Architecture Analysis Tool Suites

Ran Mo, Will Snipes, Yuanfang Cai, Srini Ramaswamy, Rick Kazman, and Martin Naedele - Central China Normal University, China; ABB Corporate Research, USA; Drexel University, USA; ABB, USA; Carnegie Mellon University, USA; University of Hawaii, USA; ABB,

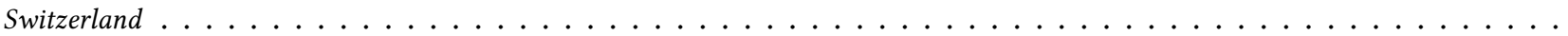

\section{New Ideas Papers}

Continuous Code Quality: Are We (Really) Doing That?

Carmine Vassallo, Fabio Palomba, Alberto Bacchelli, and Harald C. Gall - University of Zurich, Switzerland . . . . . . . . . . . .

RUDSEA: Recommending Updates of Dockerfiles via Software Environment Analysis

Foyzul Hassan, Rodney Rodriguez, and Xiaoyin Wang - University of Texas at San Antonio, USA . . . . . . . . . . . . . . . .

Delta Debugging Microservice Systems

Xiang Zhou, Xin Peng, Tao Xie, Jun Sun, Wenhai Li, Chao Ji, and Dan Ding - Fudan University, China; University of Illinois at Urbana-Champaign, USA; Singapore University of Technology and Design, Singapore . . . . . . . . . . . . . . . . . .

Personalized Teammate Recommendation for Crowdsourced Software Developers

Luting Ye, Hailong Sun, Xu Wang, and Jiaruijue Wang - Beihang University, China; Beijing Advanced Innovation Center for Big Data

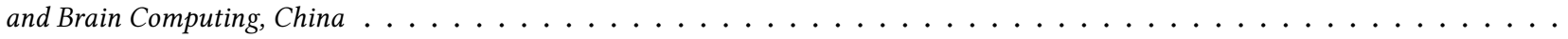

S-gram: Towards Semantic-Aware Security Auditing for Ethereum Smart Contracts

Han Liu, Chao Liu, Wenqi Zhao, Yu Jiang, and Jiaguang Sun - Tsinghua University, China; Peking University, China; Ant Financial,

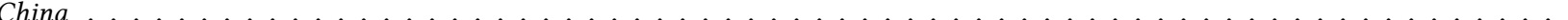

An Evolutionary Approach for Analyzing Alloy Specifications Jianghao Wang, Hamid Bagheri, and Myra B. Cohen - University of Nebraska-Lincoln, USA . . . . . . . . . . . . .

A Neural Framework for Retrieval and Summarization of Source Code Qingying Chen and Minghui Zhou - Peking University, China . . . . . . . . . . . . . . . . .

An Empirical Investigation into Learning Bug-Fixing Patches in the Wild via Neural Machine Translation Michele Tufano, Cody Watson, Gabriele Bavota, Massimiliano Di Penta, Martin White, and Denys Poshyvanyk - College of William and Mary, USA; University of Lugano, Switzerland; University of Sannio, Italy . . . . . . . . . . . . . . . . . . . . . .

Loop Path Reduction by State Pruning Jianxiong Gao and Steven S. Lumetta - University of Illinois at Urbana-Champaign, USA . . . . . . . . . . . . . . . .

node2defect: Using Network Embedding to Improve Software Defect Prediction

Yu Qu, Ting Liu, Jianlei Chi, Yangxu Jin, Di Cui, Ancheng He, and Qinghua Zheng - Xi'an fiaotong University, China . . . . . .

Towards Automating Disambiguation of Regulations: Using the Wisdom of Crowds

Manasi Patwardhan, Abhishek Sainani, Richa Sharma, Shirish Karande, and Smita Ghaisas - TCS Research, India . . . . . . . . . 


\section{Tool Demonstrations}

jStanley: Placing a Green Thumb on Java Collections

Rui Pereira, Pedro Simão, Jácome Cunha, and João Saraiva - INESC TEC, Portugal; University of Minho, Portugal; NOVA-LINCS,

Portugal; Universidade Nova Lisboa, Portugal . . . . . . . . . . . . . . . . . . . . . . . . . . . . . .

SRCIROR: A Toolset for Mutation Testing of C Source Code and LLVM Intermediate Representation

Farah Hariri and August Shi - University of Illinois at Urbana-Champaign, USA . . . . . . . . . . . . . . . . . . . .

Lightweight Source Code Monitoring with Triggr

Alim Ozdemir, Ayse Tosun, Hakan Erdogmus, and Rui Abreu - Istanbul Technical University, Turkey; Carnegie Mellon University, USA;

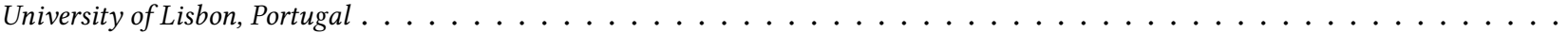

OCELOT: A Search-Based Test-Data Generation Tool for C

Simone Scalabrino, Giovanni Grano, Dario Di Nucci, Michele Guerra, Andrea De Lucia, Harald C. Gall, and Rocco Oliveto - University of Molise, Italy; University of Zurich, Switzerland; Vrije Universiteit Brussel, Belgium; University of Salerno, Italy . . . . . . . . . . .

Live Versioning of Web Applications through Refactoring Julián Grigera, Juan Cruz Gardey, Alejandra Garrido, and Gustavo Rossi - National University of La Plata, Argentina; CIC, Argentina;

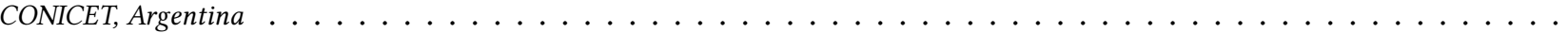

code_call_lens: Raising the Developer Awareness of Critical Code Andrea Janes, Michael Mairegger, and Barbara Russo - Free University of Bolzano, Italy $\ldots \ldots \ldots$

Code2graph: Automatic Generation of Static Call Graphs for Python Source Code Gharib Gharibi, Rashmi Tripathi, and Yugyung Lee - University of Missouri-Kansas City, USA . . . . . . . . . . . . . . .

The Electrum Analyzer: Model Checking Relational First-Order Temporal Specifications Julien Brunel, David Chemouil, Alcino Cunha, and Nuno Macedo - ONERA, France; University of Toulouse, France; INESC TEC,

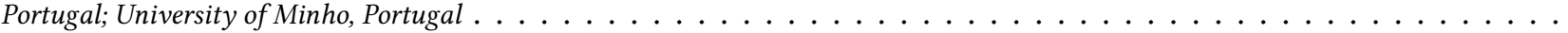

ESBMC 5.0: An Industrial-Strength $C$ Model Checker Mikhail R. Gadelha, Felipe R. Monteiro, Jeremy Morse, Lucas C. Cordeiro, Bernd Fischer, and Denis A. Nicole - University of Southampton, UK; Federal University of Amazonas, Brazil; University of Bristol, UK; University of Manchester, UK; Stellenbosch University,

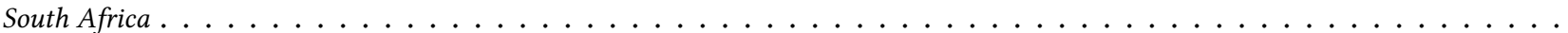

L-CMP: An Automatic Learning-Based Parameterized Verification Tool Jialun Cao, Yongjian Li, and Jun Pang - Institute of Software at Chinese Academy of Sciences, China; University of Chinese Academy of Sciences, China; University of Luxembourg, Luxembourg . . . . . . . . . . . . . . . . . . . . . .

VulSeeker: A Semantic Learning Based Vulnerability Seeker for Cross-Platform Binary Jian Gao, Xin Yang, Ying Fu, Yu Jiang, and Jiaguang Sun - Tsinghua University, China . . . . . . . . . . . . . . . . .

CPA-SymExec: Efficient Symbolic Execution in CPAchecker Dirk Beyer and Thomas Lemberger - LMU Munich, Germany . . . . . . . . . . . . . . . . . . .

CANAL: A Cache Timing Analysis Framework via LLVM Transformation Chungha Sung, Brandon Paulsen, and Chao Wang - University of Southern California, USA . . . . . . . . . . . . .

Descartes: A PITest Engine to Detect Pseudo-Tested Methods: Tool Demonstration

Oscar Luis Vera-Pérez, Martin Monperrus, and Benoit Baudry - Inria, France; KTH, Sweden

DKVF: A Framework for Rapid Prototyping and Evaluating Distributed Key-Value Stores

Mohammad Roohitavaf and Sandeep Kulkarni - Michigan State University, USA . . . . . . . . . . . . . . . . .

DroidMate-2: A Platform for Android Test Generation

Nataniel P. Borges Jr., Jenny Hotzkow, and Andreas Zeller - Saarland University, Germany . . . . . . . . . . . . . . . . .

\section{Doctoral Symposium}

\section{Session 1}

Assessing and Evaluating Functional Suitability of Software

Philipp Haindl - fKU Linz, Austria . . . . . . . . . . . . . . . . . . . . . . . .

\section{Session 2}

Automatic Mining of Constraints for Monitoring Systems of Systems

Thomas Krismayer - fKU Linz, Austria _. . . . . . . . . . . . . . . . . . . . . . . . .

Towards Automatic Restrictification of CUDA Kernel Arguments Rokiatou Diarra - University of Paris-Sud, France . . . . . . . . . . . . . . . . . . . . . . . . . . . 
A DSL for Requirements in the Context of a Seamless Approach

Florian Galinier - IRIT, France; University of Toulouse, France . . . . . . . . . . . . . . . . . . . . . .

\section{Session 3}

A Multi-objective Framework for Effective Performance Fault Injection in Distributed Systems

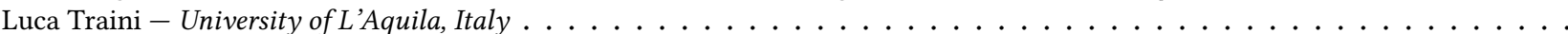

Top-Down Model-Driven Engineering of Web Services from Extended OpenAPI Models

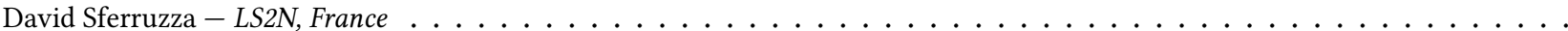

Differential Program Analysis with Fuzzing and Symbolic Execution Yannic Noller - Humboldt-Universität zu Berlin, Germany . . . . . . . . . . . . . . . . . . . . . . . . . .

Software Engineering Techniques Applied to Relational Databases Julien Delplanque - University of Lille, France; CNRS, France; Inria, France . . . . . . . . . . . . . . . . . . . . . . . .

\section{Journal-First Papers}

Automatically Quantifying the Impact of a Change in Systems (Journal-First Abstract)

Nada Almasri, Luay Tahat, and Bogdan Korel - Gulf University for Science and Technology, Kuwait; Illinois Institute of Technology, USA

Estimating the Number of Remaining Links in Traceability Recovery (Journal-First Abstract) Davide Falessi, Massimiliano Di Penta, Gerardo Canfora, and Giovanni Cantone - California Polytechnic State University, USA; University of Sannio, Italy; University of Rome Tor Vergata, Italy ～. . . . . . . . . . . . . . . . . . . . . . .

Bounded Model Checking of $\mathrm{C}_{++}$Programs Based on the Qt Cross-Platform Framework (Journal-First Abstract)

Felipe R. Monteiro, Mário A. P. Garcia, Lucas C. Cordeiro, and Eddie B. de Lima Filho - Federal University of Amazonas, Brazil; University of Manchester, UK; TPV Technology, Brazil . . . . . . . . . . . . . . . . . . . .

Understanding Semi-structured Merge Conflict Characteristics in Open-Source Java Projects (Journal-First Abstract) Paola Accioly, Paulo Borba, and Guilherme Cavalcanti - Federal University of Pernambuco, Brazil . . . . . . . . . . . . . . .

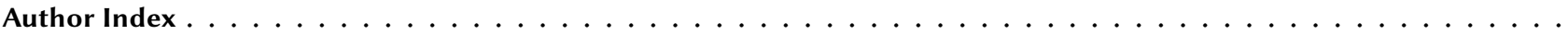

